\title{
Small-molecule inhibitor of MIF protects lupus-prone mice from kidney disease
}

An orally bioavailable small-molecule antagonist of macrophage migration inhibitory factor (MIF) has been shown to prevent the development of glomerulonephritis in two well-established mouse models of spontaneously occurring lupus. The research by Leng et al. also demonstrates that the molecule, (S,R)-3-(4hydroxyphenyl)-4,5-dihydro-5-isoxazole (ISO-1), interferes with the interaction between MIF and its receptor, CD74, by binding to the MIF tautomerase site.

As an upstream regulator in the inflammatory cascade, MIF is an attractive target for the treatment of systemic lupus erythematosus (SLE): previous studies have established that MIF is a counterregulator of glucocorticoids and also has an important role in inhibiting activationinduced apoptosis. In addition, commonly occurring high-expression MIF alleles are associated with tissue damage in several autoimmune diseases, and circulating

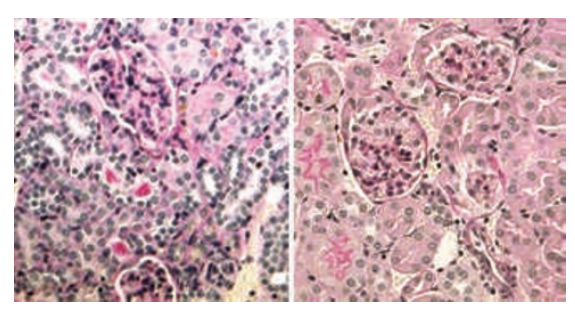

Left panel, untreated; right panel, MIF antagonist. Provided courtesy of R. Bucala.

levels of MIF with end-organ disease in patients with SLE.

In both NZB/NZW F1 and MRL/lpr mice, ISO-1 attenuated functional and histological signs of renal injury. Furthermore, inflammatory cell infiltration into the kidneys was reduced, and expression of proinflammatory cytokines and chemokines decreased. "Notably, there was little effect on the adaptive immune system, suggesting a specific, protective effect of MIF inhibitors on tissue-damaging immune responses," says Richard Bucala, corresponding author of the paper that presented the results. "This finding suggests that MIF inhibition may show selectivity with respect to an anti-disease effect, and may not be broadly immunosuppressive in clinical use." Providing support for the concept of targeting MIF, administration of a neutralizing anti-MIF antibody produced similar effects to ISO-1 in both experimental models.

Bucala contends that the studies "validate the pharmacologic targeting of MIF in autoimmune tissue damage." The translation of these findings to clinical applications will determine whether this approach is indeed effective in human autoimmune inflammatory diseases.

\section{Sarah Price}

Original article Leng, L. et al. A small-molecule macrophage migration inhibitory factor antagonist protects against glomerulonephritis in lupus-prone NZB/NZW F1 and MRL/Ipr mice. J. Immunol. 186, 527-538 (2011) 